\title{
Creatura invisibilia
}

\author{
Zur Theologie der «Mächte und Gewalten`
}

Simon Peng-Keller

Im Glaubensbekenntnis, das im Jahre 325 auf dem Konzil von Nizäa feierlich verabschiedet wurde, fällt der erste Glaubensartikel ebenso kurz aus wie im Apostolischen Glaubensbekenntnis, unterscheidet sich jedoch von diesem in einem auf den ersten Blick unerheblich scheinenden Punkt: Wird in letzterem die exklusive und alles umfassende Schöpfermacht Gottes dadurch herausgestellt, dass er als Schöpfer von Himmel und Erde bekannt wird, so ist im Nizänum stattdessen vom «Schöpfer alles Sichtbaren und Unsichtbaren» die Rede. Beide Formulierungen finden sich mit einigen Präzisierungen, mit denen ich mich noch beschäftigen werde, im Hymnus des Kolosserbriefs. Der Unterschied scheint deshalb unerheblich, weil hier wie dort offenkundig mit einer biblischen Metonymie die Allaussage von Kol 1,16a - «in ihm [Christus] wurde alles erschaffen» - veranschaulicht wird bzw. doppelt unterstrichen werden soll, dass ausnahmslos alles von allem Anfang in Christus sein Sein hat. Entgegen dem ersten Eindruck decken sich die Glieder der beiden polaren Aussagen nur annäherungsweise. So ist etwa das Unsichtbare nicht nur wie der Himmel dem menschlichen Zugriff, sondern auch seinem Erkenntnisstreben entzogen. Zudem klingt der ontologische Dual 〈Sichtbares-Unsichtbares〉 trotz biblischer Vorlage nach einem platonischen Wirklichkeitsverständnis (vgl. Phaidros 79a und Timaios 29b.92c) und wird von den Theologen der nachnizäischen Zeit zum Teil auch so interpretiert, nämlich als Differenz zwischen der sinnlichen und der geistigen Schöpfung. Die Stärke der nizäischen Formel gegenüber solchen theologischen Fortschreibungen liegt in ihrer Unbestimmtheit, die den Interpreten auf ihre biblische Fundstelle zurückverweist. Liest man sie von Kol 1,16 her, so gewinnt der fragliche und etwas blasse Ausdruck ein unerwartetes soteriologisches und dämonologisches Profil. Der Kolosserhymnus preist den erhöhten Christus nämlich als denjenigen, in dem und auf den hin alles erschaffen worden sei «im Himmel und auf Erden, das Sichtbare und das Unsichtbare, ob Throne oder Herrschaften, ob Mächte oder Gewalten.» Die meisten neueren Kommentare verstehen im Blick auf Röm 8,38f., 1 Kor 15,24, Eph 1,21 die letzten beiden Wortpaare als Näherbestimmung des «Unsichtbaren〉. Was mit 
dem Sichtbaren kontrastiert wird, ist also nicht eine höherstehende Ideenwelt oder ein neutraler Bereich der Schöpfung, der sich dem menschlichen Erkennen entzieht. Vielmehr handelt es sich um kosmische Potenzen, unsichtbare Kräfte und Schicksalsmächte, die nach dem Kolosserbrief nicht nur zur Schöpfung gehören, sondern auf Christus hin geschaffen wurden und von ihm beherrscht werden. Sie sind Verwandte des Leviathans, den zwar schon viele imaginiert (Offb 13), den aber noch niemand gesehen hat und den Gott nach einem kühnen Preisgebet dazu geschaffen hat, um mit ihm zu spielen (Ps 104,26).

Der heiklen Aufgabe einer Theologie der Mächte und Gewalten, vor die der Kolosserbrief und das Glaubensbekenntnis von Nizäa ihre systematisch interessierten Ausleger stellen, sind die folgenden Gedanken gewidmet. Um den vorgegebenen Rahmen nicht zu überschreiten, konzentriere ich mich auf den dämonologischen Aspekt und klammere die Frage nach den 〈guten Mächten〉 aus. Ich verweile zunächst bei einigen in jüngerer Zeit erkundeten Wegen, sich dem «Unsichtbaren〉 theologisch und phänomenologisch anzunähern (1.), entwickle anschliessend einige eigenen Überlegungen zum Thema (2.) und frage schliesslich nach möglichen Orientierungshilfen im Umgang mit dem 〈Unsichtbaren〉 (3.). Um zu betonen, dass ich den Leitbegriff des «Unsichtbaren` in der spezifisch biblisch-nizäischen Bedeutung gebrauche, setzte ich ihn jeweils in Anführungs- und Schlussstriche.

\section{Theologische Annäherungen an das «Unsichtbare〉}

Im deutschsprachigen Raum erschienen in den letzten Jahren gleich drei umfangreichere und im Ansatz teilweise verwandte theologische Studien, die sich als systematische Neuerschliessung des vernachlässigten theologischen Lehrstücks verstehen, das sich mit den «Mächten und Gewalten` zu befassen hat. In der im Jahre 1999 veröffentlichten Dissertation «Zwischen-Räume - Theologie der Mächte und Gewalten», die sich zur Hauptsache mit den Arbeiten von William Stringfellow und Walter Wink beschäftigt und sie miteinander zu vermitteln sucht, postuliert Thomas Zeilinger «die Notwendigkeit und die Unmöglichkeit der Wahrnehmung der Mächte» (359). Die Theologie und die Verkündigung sind nach Zeilinger vor die schwierige Aufgabe gestellt, die Wahrnehmung der unsichtbaren Mächte〉 durch christlich-mythologische Rede und 
kreative Imagination $\mathrm{zu}$ ermöglichen, ohne sie mythisch $\mathrm{zu}$ substantialisieren. Haben sie doch keinen eigenständigen ontologischen Status, sondern sind «Zwischenmächte» zwischen Gott und Mensch, die ihre wirksame Pseudo-Wirklichkeit der Verkehrung der guten Schöpfung verdanken. Zeiliger plädiert im Anschluss an Stringfellow dafür, die Mächte einerseits liturgisch-exorzistisch «zurechtzuweisen〉 und ihnen andererseits durch sozialpolitisches Engagement die Stirn zu bieten.

Ähnlich kämpferisch gibt sich Thomas Rusters 2005 erschienene «Himmelslehre» mit dem eingängigen Titel «Von Menschen, Mächten und Gewalten». Nach Ruster haben das neuzeitliche Denken und die postmoderne Himmelsfrömmigkeit die beiden theologisch wichtigen Unterscheidungen zwischen Gott und Himmel bzw. Himmel und Erde verwischt und sich dadurch die Möglichkeit verbaut, die «Mächte und Gewalten〉 erkennen und bekämpfen zu können. Wer den Himmel als den unzugänglichen und unverfügbaren Bereich der Welt verleugne oder mit Gottes Transzendenz verwechsle, verkenne die Mächte, die ihr Wesen im Verborgenen viel besser treiben können, ebenso wie das Wirken Gottes, das sich diesem Treiben entgegenstellt. Im Gegensatz zu Zeilinger, der auf die Beteiligtenperspektive des (Un-)Glaubens und die Unbeobachtbarkeit der Mächte abhebt, bezieht Ruster die Rede von den Mächten und Gewalten auf «Phänomene, die in der Reichweite heute zugänglicher theoretischer Anschauungen» liegen (17). Die verschleiernde Rede von ‘höherer Gewalt`, die analysierbare Zusammenhänge und kontingente Gegebenheiten in einen unverfügbaren Bereich entrücke, sei um Gottes und des Menschen willen $\mathrm{zu}$ entmystifizieren und durch wissenschaftliche Beschreibungen $\mathrm{zu}$ ersetzen. (Was Ruster allerdings nicht daran hindert, abschnittweise selber ins mythologische Sprachspiel zu wechseln, und beispielsweise $\mathrm{zu}$ beschreiben, mit welchen Gefühlen die «Mächte〉 einer Eucharistiefeier beiwohnen, die sie schliesslich "wie geprügelte Hunde» verlassen müssen). Als Inbegriff von entfesselten Mächten, die Ruster im Anschluss an Niklas Luhmann als selbstzerstörerische Eigendynamik autonomisierter Funktionssysteme begrifflich zu fassen versucht, erscheint hier die globalisierte (freie) Marktwirtschaft, die die Menschheit und die Ökosysteme der Erde auf den totalen Kollaps zusteuere. Wie Zeilinger setzt auch Ruster im Kampf gegen die «unsichtbaren Mächte` auf die exorzistische Kraft der Liturgie und - mehr noch - auf den sozialpolitischen Widerstand, den er als gerechtes Handeln nach der Tora profiliert. 
Deutlich zurückhaltender mit Anweisungen zu christlichem Leben und liturgischem Tun ist Martin Hailers ein Jahr später erschienene Habilitationsschrift «Gott und die Götzen. Über Gottes Macht angesichts der lebensbestimmenden Mächte». Sie fragt nach den theologischen Konsequenzen des Bekenntnisses, dass die unsichtbaren Mächte Gott unterworfen sind und durch ihn überwunden werden. Im Anschluss an Karl Barth beschreibt Hailer die fraglichen Kräfte als «herrenlose Gewalten, die als Exponenten des Nichtigen eine eigentümliche Zwischenexistenz zwischen Gott und Schöpfung einnehmen» (252). Als Trugbilder des Unglaubens suggerieren sie ihre Absolutheit und Unausweichlichkeit und verdunkeln das Welt- und Gottesverständnis des Menschen. Ihrer Unsichtbarkeit als derVerschleierung, die sie umgibt und die sie bewirken, kommen deshalb auch theoretisch-analytische Versuche der Sichtbarmachung nicht bei. Ihr nichtiges und gottwidriges Wesen offenbart sich nur dem gläubigen Blick, der nach Karl Barth nur kurz und unwirsch auf ihnen ruhen soll. Im Kampf gegen die Mächte setzt Hailer deshalb auf die desuggestive Kraft des Glaubens und die exorzistische Gegenmacht seiner Bilderwelt und Narrationen: «Was im theologisch präzisen Sinne entmythologisiert, ist nicht nur die Entlarvung lügenhafter Gespinste. Tatsächlich entmythologisierend wirkt das Heimischwerden in einer anderen Geschichte, als sie von den Mächten aufgedrängt wird, das Bewohnen anderer Bildwelten als diejenigen, die sie vorgaukeln. Der eschatologische Realismus als Beschreibung des Widerlagers gegen die Mächte ist nicht bildlos, er lädt vielmehr zum Bewohnen der Geschichts- und Bildwelt des Evangeliums ein» (392).

Im Anschluss an die Studien von Zeilinger, Ruster und Hailer wende ich mich in den nächsten beiden Abschnitten zwei Fragen zu, die von ihnen unterschiedlich beantwortet werden: Inwiefern sind die «Mächte und Gewalten` als unsichtbar zu qualifizieren (2.) und wie lässt sich mit ihrer Präsenz leben, ohne von ihnen beherrscht zu werden (3.)?

\section{Unsichtbarkeit und Phänomenalität der «Mächte und Gewalten>}

Die zurückhaltende Formulierung des Nizänums erlaubt es heutiger Theologie, guten Gewissens auf eine ausgefaltete Angelologie und Dämonologie zu verzichten. Will sie jedoch den Topos nicht gänzlich anderen überlassen, so bietet es sich an, von der metaphorischen 
Prägnanz auszugehen, die der Rede von unsichtbaren «Mächten und Gewalten offenkundig zu eigen ist. Die betreffenden Mythologeme, die weitgehend dem «semantischen Feld der politischen Herrschaft» entnommen sind (M. Wolter), zeichnen sich dadurch aus, dass sie etwas indizieren, was sich der Menschen kollektiv bemächtigt, ohne sich ihnen $\mathrm{zu}$ entschleiern. Sie stehen darin in einem deutlichen Kontrast zur Rede vom Heiligen Geist, die diesem zwar ebenfalls Unsichtbarkeit und Wirksamkeit zuschreibt, diese jedoch anders qualifiziert. Die ontologische Differenz zwischen Gottes Geist und den 〈Mächten〉 könnte kaum grösser sein, sind doch letztere in einem theologisch präzisen Sinn nichtig. Als «Mächte〉 existieren sie nur kraft der durch sie übermächtigten Menschen. Als solche sind sie so wirksam wie unsichtbar. Denn sie leben davon, nicht als gottwidrige Mächte erkannt zu werden. Soweit sie im Glauben erkannt werden, depotenzieren sie sich $\mathrm{zu}$ mehr oder weniger menschlich beeinflussbaren und insofern nicht mehr völlig unsichtbaren Wirkzusammenhängen.

Das zweite Jesajabuch beschreibt die Unsichtbarkeit der 〈Mächte〉 und ihre entdämonisierende Sichtbarwerdung auf eindingliche Weise. Einerseits gelte: «Wer sich zu seinen Göttern bekennt, sieht nichts, ihm fehlt es an Einsicht» (44,9). Er sieht nicht, was dem Propheten und seinen Adressaten offenkundig ist und verfällt gerade dadurch der Faszination seiner Imaginationen und Fabrikationen. Auch in der Perspektive, die der prophetische Text eröffnet, treten keine mythischen Unwesen auf.Vielmehr sieht man nichts anderes als einen in seiner Selbstbezüglichkeit gefangenen Menschen: «Der Zimmermann hat die Richtschnur ausgespannt, unermüdlich hat er mit dem Griffel vorgezeichnet, es mit den Schnitzmessern ausgeführt (...). Er ist gegangen, um sich Zedern zu fällen, und hat eine Steineiche genommen oder eine Eiche, und unter den Bäumen des Waldes hat er ihn kräftig werden lassen für sich. Er hat Lorbeer gepflanzt, und der Regen hat ihn gross gemacht, und dann hat er einem Menschen als Brennholz gedient. Und dieser hat davon genommen und hat sich damit gewärmt. Er zündet es an und backt Brot! Er macht einen Gott daraus! Und schliesslich hat er sich niedergeworfen, hat es zum Bild gemacht und sich vor ihm verbeugt» (44,13-15). Die prophetische Götzenkritik arbeitet mit Spott und einer deskriptiven Genauigkeit, die spätere philosophisch-wissenschaftliche Entzauberungsmethoden vorwegnimmt. Sie unterscheidet sich von diesen darin, dass sie, wie der Hymnus des Kolosserbriefs, die Unsichtbarkeit der 〈Mächte〉 auf die Unsichtbarkeit Gottes bezieht und so das Tun des Götzenbilderfabrikanten nicht nur als 
Aberglauben und Magie diagnostiziert, sondern als Unglauben entlarvt, der sich an die trügerische Evidenz des Sichtbaren hält. Anders als etwa das letzte Buch der Bibel, das eine dramatische imaginative Sichtbarmachung der unsichtbaren Mächte inszeniert, wählt das zweite Jesajabuch die Strategie der desuggestiven Distanznahme, in der sich die Faszinationskraft, die das merkwürdige und zugleich banale Tun motiviert, in Nichts auflöst.

Bei aller theologisch herauszuarbeitenden Nichtigkeit der Mächte ist ihre Verführungskraft ernst zu nehmen, was u.a. bedeuten kann, sich um ein genaueres Verständnis der hier wirksamen Mechanismen zu bemühen. Der jesajanische Spott und der deuteropaulinische Hymnus haben diesbezüglich wenig beizutragen, lassen sie es doch im Dunkeln, auf welchen Wegen die unnsichtbaren Mächte` die sichtbare Welt durchformen. Versucht man dieses Dunkel etwas aufzuhellen, so lassen sich die Verführungsmechanismen, die hier im Spiel sind, dreifach spezifizieren.

Der Autor von Jes 44 scheint die kollektive Einbindung des von ihm porträtierten Götzendieners bewusst auszublenden, um die Absurdität der beschriebenen Tätigkeit herauszustellen. Will man jedoch verstehen, was die Macht der «unsichtbaren Mächte` ausmacht, genügt es nicht, einem einzelnen Bildschnitzer oder einem VoodooPriester bei der Arbeit zuzuschauen. Die «Mächte und Gewalten〉 herrschen über ganze Menschengruppen und werden biblisch nicht einzelnen Besessenen zugeordnet. Man kann sich fragen, inwiefern die 〈Mächte〉 und 〈Gewalten〉 der Höhe und der Tiefe, von denen Paulus in Röm 8,28f. spricht, eine metaphorische Variation des Sprechens von der herrschenden〉, «täuschenden〉 und «tötenden〉 Sünde darstellt, von der die vorausgehenden Kapitel des Briefes handeln. Die Verbindung zwischen den beiden Aussagereihen bleibt merkwürdig lose. Offenkundig ist es metaphorologisch bedeutsam, wenn im Gegensatz zum Singular der 〈herrschenden Sünde` von den «Gewalten` im Plural gesprochen wird. Als Konkretisierungsformen der Sünde treten sie in der Mehrzahl auf. Ihre Angewiesenheit auf ein bestimmtes Kollektiv begrenzt zugleich eine beliebige Pluralisierung. Nach Karl Barth finden sich die ‘herrenlosen Mächte> v.a. in vier Gestalten: in politischen Absolutismen, in der Faszination von Besitz und Geld, in geistigen Wirkzusammenhängen wie Ideologien, Weltanschauungen, nicht-musealen Mythen u.s.w. und schliesslich in der negativen Eigendynamik von Technik und kulturellen Strömungen, die Barth den «chtonischen Mächten» zuordnet.

Das zweite Merkmal, durch das sich die Verführungskraft der Mächte charakterisieren lässt, unterläuft Barths Unterscheidung 
zwischen geistigen und chtonischen Mächten. Auch die kollektiven Kräfte, die in Ideologien und Weltbildern wirksam sind, suchen bekanntlich ihre aufmerksamkeitslenkenden und einprägsamen Symbole, Rituale und Ausdrucksformen. Sie greifen dazu auf bestehende Sprachspiele und Symbolsysteme zurück und führen manchmal zur Bildung von Subkulturen, die für den Aussenstehenden ebenso merkwürdig wirken wie das von Jesaja beschriebene Tun des Statuenschnitzers. Fiktionen sind die «Mächte und Gewalten〉 jedoch nicht erst in Gestalt solcher Materialisierungen und Ausgestaltungen, sondern schon dadurch, dass sie «Protuberanzen des dem Menschen als Gottes Geschöpf verliehenen Vermögens» darstellen (K. Barth). In ihrer passiven Genesis aus den verschiedenartigen menschlichen Vermögen sehe ich ihr drittes Merkmal. Im Bild gesprochen leben die «Mächte und Gewalten` auf ungleich innigere Weise, als es parasitäre Pflanzen und Tiere im Verhältnis zu ihren Wirten tun, aus dem Vorrat menschlicher Kräfte und Möglichkeiten. Als vom Menschen abkünftige herrenlose Mächte〉 nutzen sie seine Imaginations-, Überzeugungs- und Gestaltungskraft, kultivieren seine Wünsche und beuten seine Sehnsucht aus. Sie gehören, so gottähnlich sie sich auch gegenüber ihm gebärden mögen, zum Menschen, dem sie entlaufen sind.

\section{Vom Umgang mit flüchtigen Drachen}

Die Metapher der entlaufenen und nun herrenlosen Gewalten beinhaltete in der traditionellen christlichen Dämonologie im Gegensatz zur eben vertretenen These meist die Vorannahme, es handle sich hier um geistige Wesenheiten, die nicht dem Menschen, sondern Gott entlaufen sind und seiner Herrschaft trotzen. Der Epheserbrief sieht es aber bekanntlich anders, zumindest was das Herrschaftsverhältnis betrifft, wenn er den erhöhten Christus als denjenigen verkündet, dem alle «Fürsten und Gewalten, Mächte und Herrschaften» unterworfen sind $(1,21 \mathrm{f}$.). Wer den Glauben an das noch unsichtbare aber schon gegenwärtige Reich Gottes teilt, kann nach Evagrios Pontikos die unheimlichen Wesenheiten getrost mit Nichtbeachtung strafen: «Was aber jenen «flüchtigen Drachen〉 betrifft, der euch bedrängt, so denkt nicht an ihn und haltet ihn nicht für etwas und fürchtet euch nicht vor ihm. Er ist nämlich ein flüchtiger Sklave, der schlecht gelebt hat und seinem Herrn entlaufen ist. Gebt ihm keinen Raum bis zum Tode! (...) Ist nicht er es, der von unserem Herrn die Schweineherde erbat und der sich zuvor 
die Güter des Hiob ausbat? Wenn er nicht die Macht hat, sich den Schweinen und Eseln zu nähern ohne die Zustimmung des Gebers, wie sollte er dann Macht haben über das Ebenbild Gottes? Wir selbst unterwerfen uns den Dämonen durch unseren Unglauben! (...) Der Drache weiß nur zu drohen. (...) Darum überlasst ihm nicht eure «Behausung», denn ihr seid diese Niederlage nicht wert» (Brief 28). Der byzantinische Theologe Evagrios betreibt im bildungsfeindlichen Milieu des koptischen Mönchtums, in dem er seine letzten Lebensjahre verbringt, eine asketisch-mystisch geprägte Form christlicher Entmythisierung. Er weist darauf hin, dass man den «Mächten` nicht nur durch Unaufmerksamkeit, sondern auch durch ein Zuviel an Aufmerksamkeit erliegen kann. Wer ihnen Beachtung schenkt, steht bereits in ihrem Wirkfeld. Nicht an die herrenlosen Gewalten zu denken und sie nicht für etwas halten, bedeutet für ihn allerdings nicht, dem keine Aufmerksamkeit zu schenken, was sie im und zwischen den Menschen bewirken. Evagrios ermuntert vielmehr zu einer genauen Selbstbeobachtung und ordnet selber die negativen Gedanken in ein nachhaltig wirksames heuristisches Raster. Die dämonischen Mächte, so sein Grundgedanke, haben nämlich im Unterschied zu Gottes Geist keinen unmittelbaren Zugang zum menschlichen Herz, sondern wirken mittels Gedanken (logismoi), die sie im Geist des Menschen zur Ausbildung bringen. Es gilt diese Phantasien des Unglaubens frühzeitig wahrzunehmen und zu identifizieren, um sie dadurch um ihre Wirkmacht zu bringen. Es ist allerdings einzuräumen, dass Evagrios' individualpsychologischer Zugang blind ist für die transpersonale Eigendynamik der herrenlosen Mächte), die Zeilinger, Hailer und Ruster in der globalen Aufrüstung oder den Finanzmärkten am Werke sehen. Die «Mächte» haben seit dem 4. Jahrhundert ihre Überredungskünste und Überzeugungstechniken verfeinert und Schritt gehalten mit der medialen und ökonomischen Globalisierung, so wie auch ihren christlichen Verächtern heute schärfere diagnostische Instrumente zur Verfügung stehen als die stoische Psychologie, auf die Evagrios zurückgriff. Aus dessen Anweisungen für das Leben inmitten von ‘flüchtigen Drachen〉 dürfte sich jedoch diejenige bleibend bewähren, ihre durch Glanz und Schatten aufmerksamkeitsheischende Präsenz mit achtsamer Nichtbeachtung zu strafen und ihre achtungsgebietende und ehrfurchtserregende Selbstpräsentation als Einbildung des Unglaubens zu durchschauen. Die creatura invisibilia, die ins Sichtbare hineindrängt und sich hinter ihm verbirgt, ist glaubend von der invisibilia Dei (Röm 1,20) zu unterscheiden, die in seiner Schöpfung widerglänzt als Vorschein einer Wirklichkeit, die im Werden ist. Das 
berechtigt die Bekenntnisse von Nizäa und Konstantinopel, nur am Rand und leicht überhörbar von den «unsichtbaren Mächten〉 zu reden und sie als zunehmend Entmächtigte der kommenden Präsenz des Erhöhten unterzuordnen.

- Dr. Simon Peng-Keller ist wissenschaftlicher Mitarbeiter am Lehrstuhl für Fundamentaltheologie und Ökomene an der Universität Fribourg. 